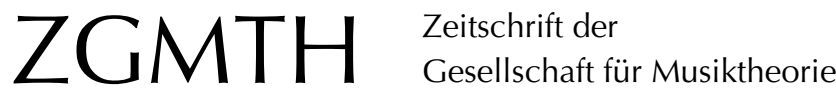

Quint, Johannes (2010): Unendliche Skepsis: Offene Form in Chopins Mazurken. ZGMTH 7/3, 255-266. https://doi.org/10.31751/603

(C) 2010 Johannes Quint

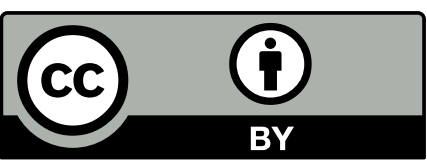

Dieser Text erscheint im Open Access und ist lizenziert unter einer Creative Commons Namensnennung 4.0 International Lizenz.

This is an open access article licensed under a

Creative Commons Attribution 4.0 International License.

veröffentlicht / first published: 22/12/2010 zuletzt geändert / last updated: 21/05/2011 


\title{
Unendliche Skepsis: Offene Form in Chopins Mazurken
}

\author{
Johannes Quint
}

Chopins Mazurken sind wegen ihrer harmonischen Phantastik berühmt. Seltener wurden sie mit Blick auf ihre oftmals experimentelle Form beschrieben. Im Beitrag wird die Schlussgestaltung einiger dieser Werke fokussiert. Dabei zeigen sich ganz unterschiedliche Varianten einer >offenen Form‘, die es ermöglichen, eine Querverbindung zwischen konkreten kompositionstechnischen Details und Kernpunkten frühromantischer Philosophie (Novalis und Friedrich Schlegel) herzustellen.

\section{Unendlichkeit}

Frédéric Chopins Mazurka op. 7,5 ist in formaler Hinsicht ein Grenzfall. Nach einem kurzen Begleitvorlauf besteht das Stück aus zwei Achttaktern gleichen Inhalts, der erste in C-Dur, der zweite in der Dominanttonart G-Dur.
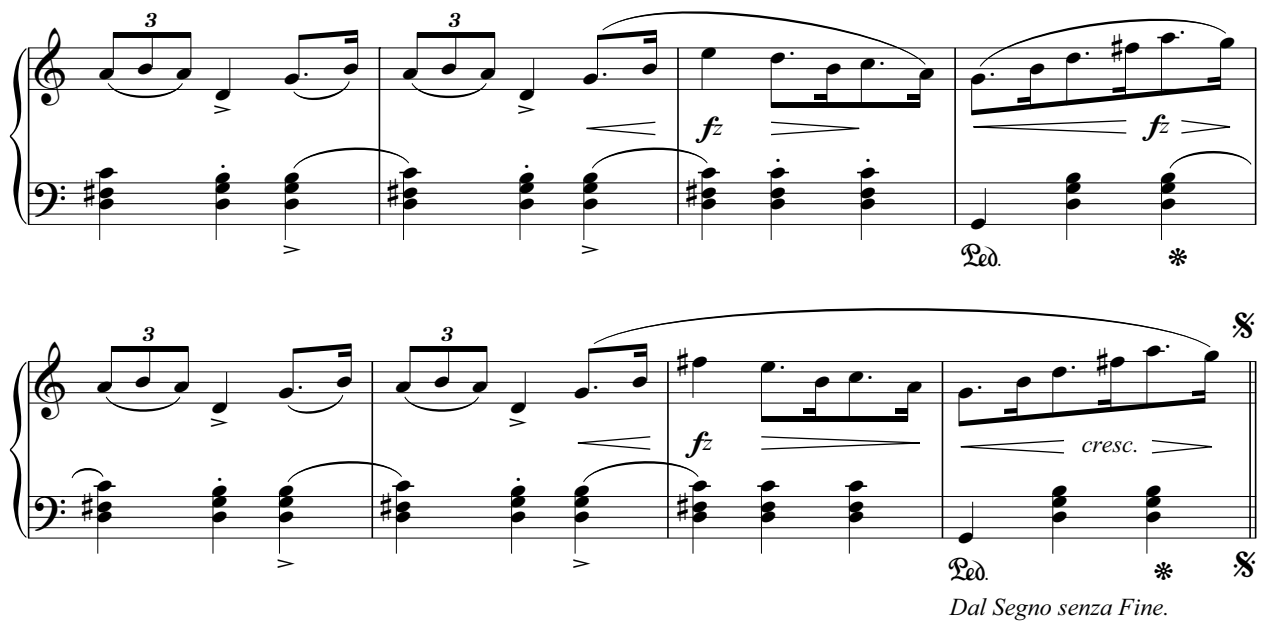

Beispiel 1: Frédéric Chopin, Mazurka op. 7,5, Schluss

Am Ende des Stückes steht die Anweisung: „Dal segno sine fine«. Man kann darin eine Reminiszenz an den Oberek (einen rauschhaft-schnellen polnischen Tanz) sehen, doch 
wäre es naiv zu glauben, Chopin hätte hier nicht auch die formale Paradoxie mitreflektiert, zumal op. 7,5 das Schlussstück des Zyklus bildet: Nähme man die Anweisung beim Wort, ergäbe sich eine Form, die niemals zu einem Ende kommt.

Es gibt kein zweites Beispiel, in dem Chopin Unendlichkeit so wörtlich umsetzt, doch auf subtile Weise thematisieren viele Mazurken die Idee einer offenen Form. Obwohl, oder vielleicht gerade weil Mazurken in der Regel sehr einfach gebaut sind - fast alle bestehen aus dreiteiligen Reprisenformen - zeigen sich hier vielfach und in ganz unterschiedlicher Weise Chopins Skrupel gegen konventionelle Schlusswendungen und Schlusswirkungen. Diese Tendenz zur Entgrenzung verweist auf ein generelles Thema der Romantik, ein Thema, das sich auch in frühromantischer Philosophie findet. So lautet ein Fragment von Novalis:

Wir sind aus der Zeit der allgemeingeltenden Formen heraus. ${ }^{2}$

Einer der Hauptangriffspunkte von Novalis und des frühen Friedrich Schlegel ist die unreflektierte Verwendung abgeschlossener Denksysteme:

Das eigentliche Philosophische System muss Freyheit und Unendlichkeit, oder, um es auffallend auszudrücken, Systemlosigkeit, in ein System gebracht seyn. ${ }^{3}$

Wer ein System hat, ist so gut geistig verloren, als wer keins hat. Man muß eben beides verbinden. ${ }^{4}$

Was sich hier ankündigt, ist die Auflösung des herkömmlichen, auf Geschlossenheit zielenden Werkbegriffes. Dergleichen geschieht in Chopins Experimenten: Sie widerspiegeln dieselbe geistige Strömung und antizipieren damit eine insbesondere für das 20 . Jahrhundert bestimmende Entwicklung, die bis in unsere Gegenwart hineinwirkt.

\section{Offene Schlüsse}

Schlegel betont mit Bedacht die Paradoxie von Systemlosigkeit als System. Auf die Ebene künstlerischer Form übertragen: Eine Komposition hat notwendigerweise einen Anfang und einen Schluss. Gleichwohl kann - als Moment des Poetischen - eine offene Form suggeriert werden, wenn das ıäußere s Schließen umschlägt in ein sinneres`Weiterklingen. Chopins Schlussgestaltungen zeigen nun auf unterschiedliche Art, wie diese Dialektik auskomponiert werden kann. Betrachten wir dazu drei Beispiele:

1 Vgl. Lissa 1973.

2 Novalis, Fragmente 1998, 649, Nr. 479.

3 Novalis 1965, 288.

4 Schlegel 1963, 80. 
Op. 24,3: Verklingen
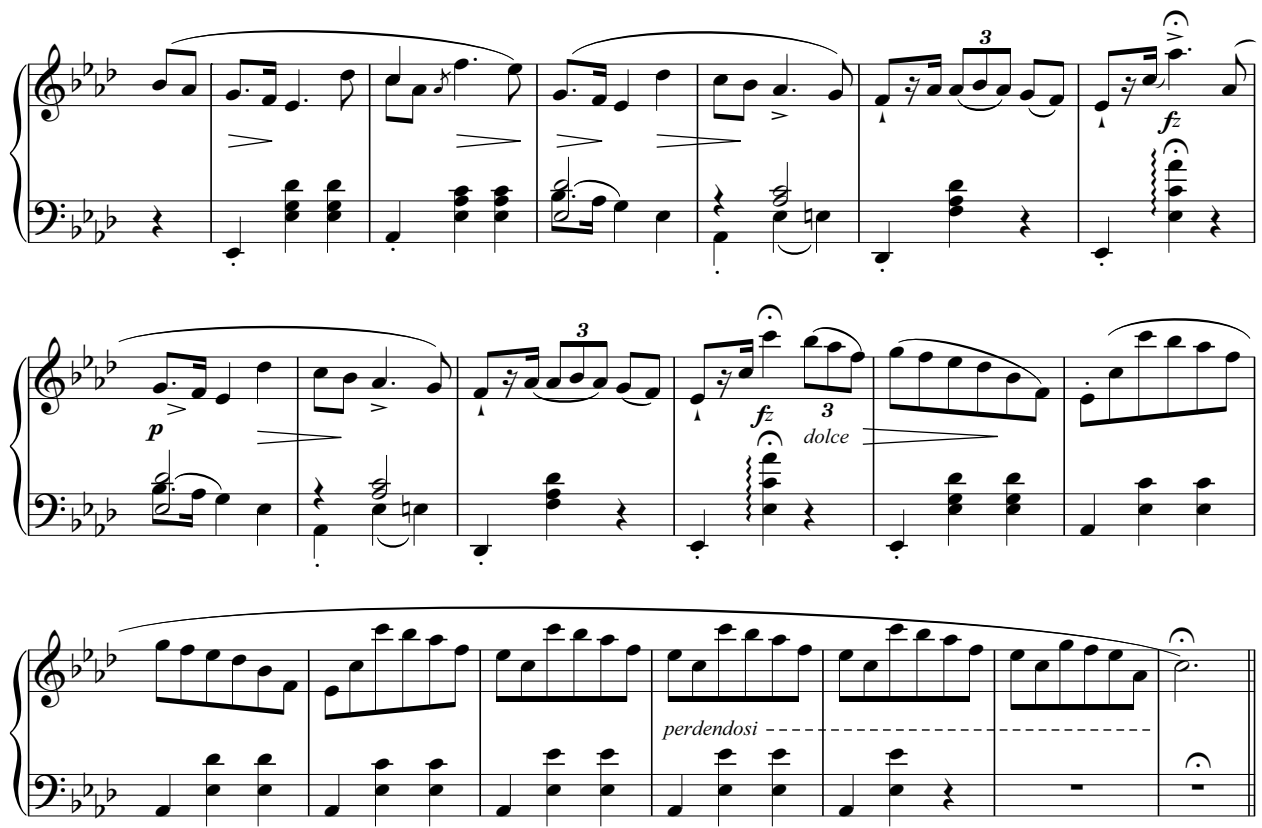

Beispiel 2a: Frédéric Chopin, Mazurka op. 24,3, Schluss

Ähnlich gehalten ist der Schluss von Bed ich Smetanas Moldau, der das Sich-Entfernen des Flusses malt:

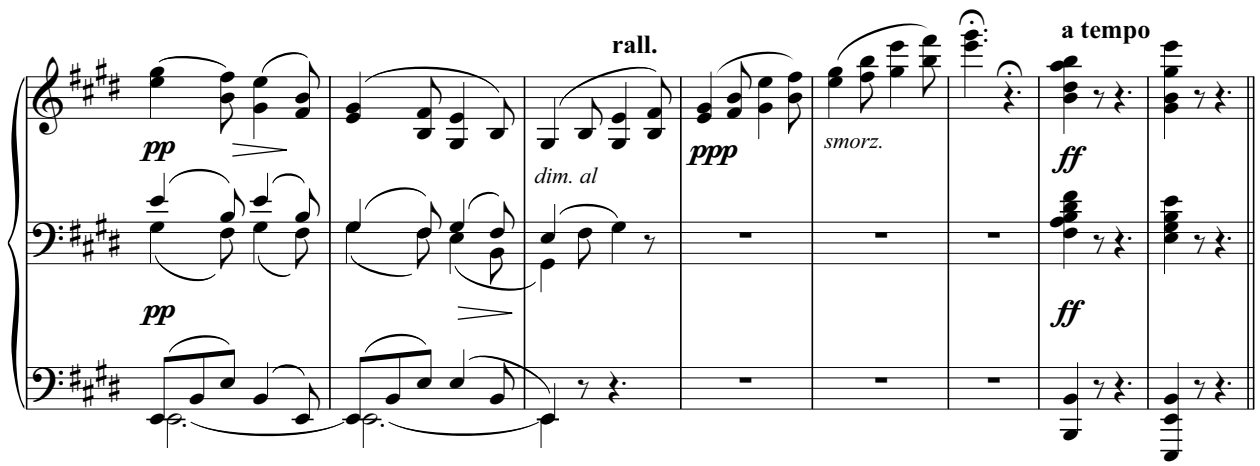

Beispiel 2b: Bed ich Smetana, Die Moldau, Schluss, Particell

Allerdings: Während Smetana an den offenen Schluss gewaltsam eine brutale Kadenz anhängt, motiviert Chopin mit der Andeutung einer D-T-Wendung den Schluss sehr fein 
als Verklingen. Man vergleiche Chopins Version mit einem fiktiven Schluss, durch den die Musik schlicht ausgeblendet wird:

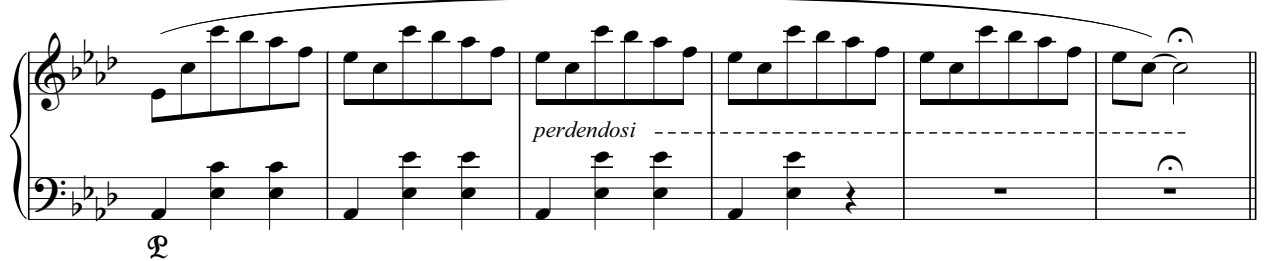

Beispiel 2c: Frédéric Chopin, Mazurka op. 24,3, rekomponierte Variante des Schlusses

Op. 30,1: Sehnsucht
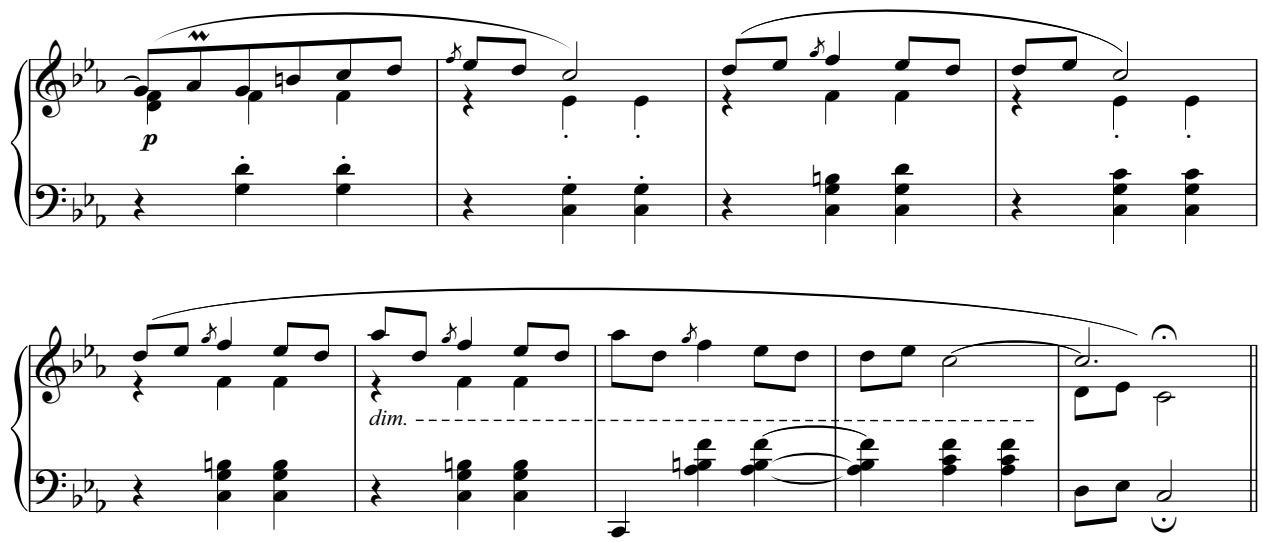

Beispiel 3a: Frédéric Chopin, Mazurka op. 30,1, Schluss

Auch die Mazurka op. 30,1 endet offen. Doch ist die Wirkung hier eine ganz andere als bei op. 24,3. Weil Chopin öffnende und schließende Momente gegeneinander ausbalanciert ergibt sich eine Ambivalenz: Der Hörer pendelt zwischen Schlussgefühl und Fortsetzungserwartung - ein Spiel, ganz im Sinne der Philosophie Friedrich Schlegels. Analysieren wir die widerstrebenden Momente genauer und werfen zunächst einen Blick auf die Großform. Die Mazurka ist als Reprisenform gebaut, mit der typischen Anlage:

A: $8+8$ (Doppelperiode) | B: $8+12$ (erweiterte Doppelperiode) | Reprise von A

In der zweiten Periode der Reprise wird der Nachsatz durch eine Verlängerung des Vordersatzes ersetzt. Das erscheint mit Blick auf den zu erwartenden Schluss durchaus konsequent, moduliert doch die im A-Teil wörtlich wiederholte Ursprungsperiode (Mazurka op. 30,1, T. 1-8). Der erweiterte Vordersatz verlängert die Haupttonart, und dadurch, dass er auf einem Tonikaorgelpunkt ruht, deutet Chopin ab Takt 49 die Formfunktion 
¿Codar an. Wodurch ergibt sich aber nun der seltsame Schwebezustand, der sich am Schluss einstellt? Zuerst einmal ist dafür die linke Hand verantwortlich: Im vorletzten Takt löst sich der verminderte Septakkord über dem Orgelpunkt nicht in die Tonika c-Moll, sondern in einen f-Moll-Sextakkord (oder Quartsextakkord, wenn man das tiefe $c$ noch mithört) auf. Das hat nun zwei Konsequenzen:

\section{Harmonisch}

Um eine reguläre Schlusskadenzierung zu erreichen, muss der f-Moll-Sextakkord als Vorhaltsbildung plagal in die Tonika aufgelöst werden: Das reguläre Harmonieschema sähe folgendermaßen aus:

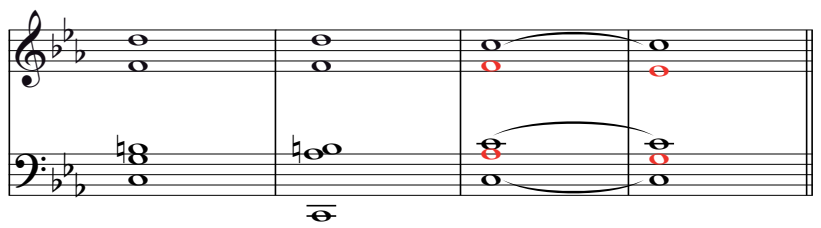

Beispiel 3b: Frédéric Chopin, Mazurka op. 30,1, Schluss, Harmonieschema

\section{Formal}

Aus dem Vorhaltsakkord in Takt 52 ergibt sich ein zusätzlicher Takt, der in der Reprise aus der 8+8-Doppelperiode des A-Teils eine 8+9-Taktgruppe macht. Durch diese Asymmetrie ist der Reprise von vorneherein die Chance genommen ırund zu schließen. Die Pointe des letzten Taktes liegt nun darin, dass er die erwartete Schlussbildung vollzieht, aber zugleich wieder zurücknimmt. Durch die Einstimmigkeit wird eine plagale Kadenz und damit die Schlusstonika angedeutet, die charakteristische Quartauflösung auch tatsächlich vollzogen, aber der vollständige Klang der Tonika fehlt.

Auch das ein romantischer Topos: die Empfindung des Mangels, der Sehnsucht.

\section{Op. 41,3: Fragment}

Die Mazurka op. 41,3 endet mit einem der verblüffendsten Schlüsse, die Chopin komponiert hat (Beispiel 4a).

Chopin komponiert hier ein echtes Fragment, indem er den erwarteten letzten Achttakter nach sechs Takten abbrechen lässt. Doch schauen wir auch hier genauer hin: Dieser Achttakter, mit dem bereits der A-Teil schließt, moduliert von As-Dur nach c-Moll. Dabei ist die Reharmonisierung der Zählzeit 3 im zweiten Takt des jeweiligen Viertakters entscheidend: Beim ersten Mal führt eine Zwischendominante in die Subdominante von As-Dur, beim zweiten Mal wird das $c^{2}$ in der Melodie mit einem halbverminderten Septakkord in Terzquartakkordstellung harmonisiert, der als Subdominante einer Kadenz in c-Moll fortgeführt wird. Während am Ende des A-Teils die Modulation genutzt wird, um eine kurze sMitte in C-Dur anzuschließen (T. 41ff.), ist in der Reprise eine Modifikation 

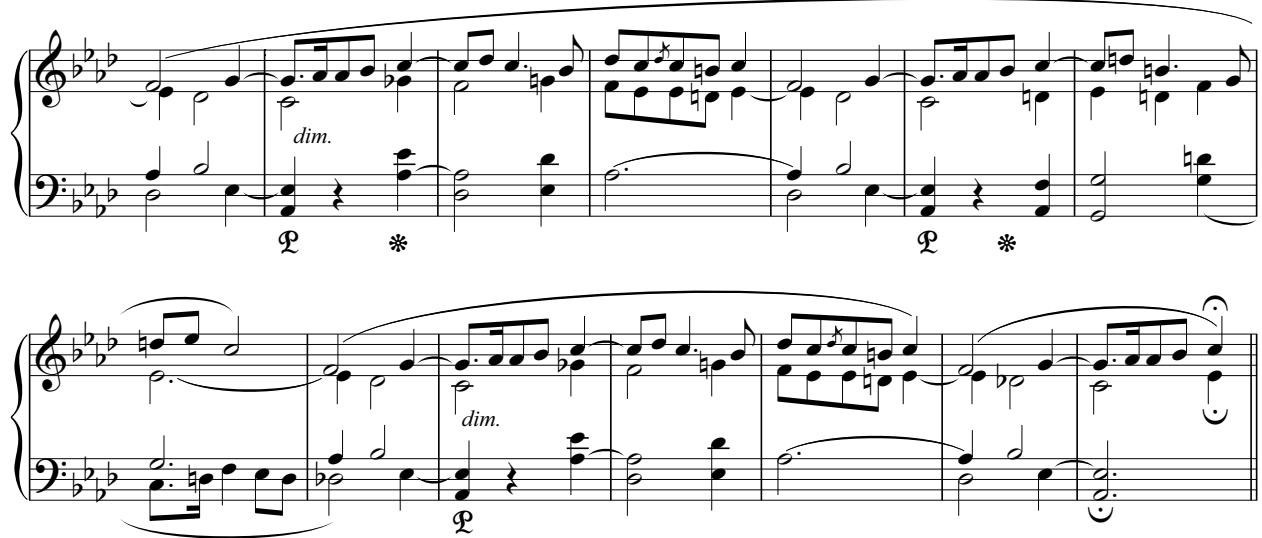

Beispiel 4a: Frédéric Chopin, Mazurka op. 41,3, Schluss

dieser Stelle erforderlich, damit in der Haupttonart geschlossen werden kann. Versuchen wir einen regulären Schluss:

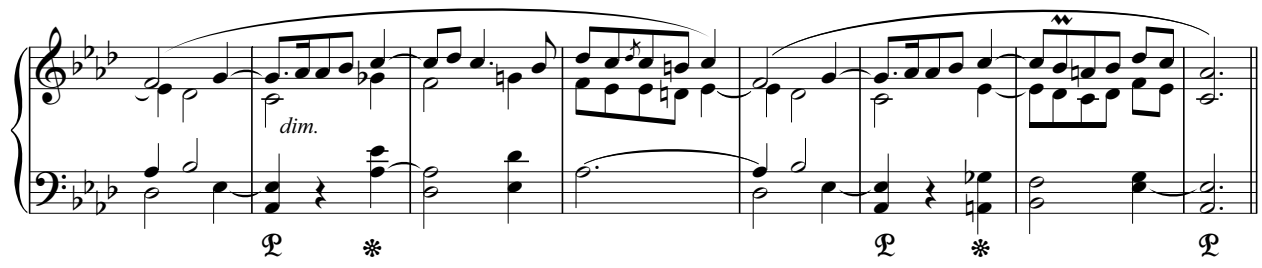

Beispiel 4b: Frédéric Chopin, Mazurka op. 41,3, Schlussachttakter, Rekomposition

Die Rekomposition verdeutlicht, dass die Pointe des originalen Schlusses im Verhältnis von Form und Stufengang liegt. Auf dem Weg, den die Taktgruppe nimmt, nutzt Chopin sozusagen eine sgünstige`Stelle, um das Stück in As zu beschließen. Die Anfangstonika der Kadenz wird dabei überraschend zur Endtonika erklärt; das $C^{2}$ in der Melodie erfährt - anders als bisher - keine Reharmonisation, sondern wird Terz im beibehaltenen As-Dur-Akkord. Allerdings erscheint der Schluss im sechsten statt im achten Takt der Taktgruppe und damit in formaler Hinsicht zu früh. Hinzu kommt, dass der Schlussklang auf dem dritten Viertel, der leichten Zeit des Taktes erreicht wird. Die Musik wirkt wie abgebrochen - es entsteht der Eindruck des Fragmentarischen.

\section{Exkurs: Bezüge zur Klassik}

Der differenzierte Umgang mit Schlusswirkungen ist keine Erfindung der Romantik. Er findet sich zu allen Zeiten der Musikgeschichte, wann immer sich Schlusswendungen 
konventionell verfestigen. Doch erst in der Klassik beginnt die Gestaltung der Schlüsse die Bedeutung zu bekommen, die dann die Formen Chopins so auflädt. Insbesondere in Joseph Haydns Streichquartetten finden sich bemerkenswerte Grenzgänge in Bezug auf geschlossene und offene Formen. Zwei Beispiele dazu: Das Menuett aus Haydns op. 33,5 schließt folgendermaßen:
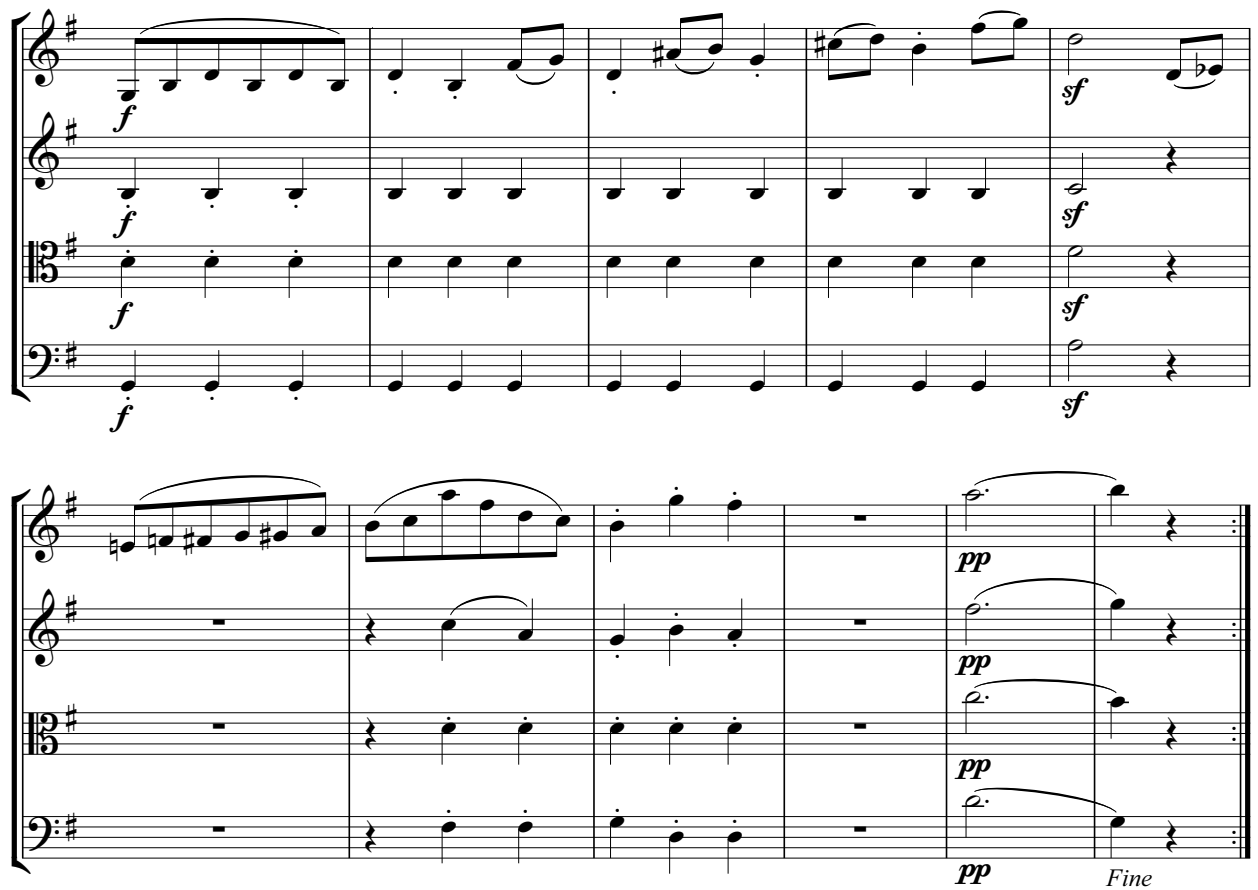

Beispiel 5: Joseph Haydn, op. 33,5, Menuett, Schluss

Auch hier gibt es eine Ambivalenz von Offenheit und Geschlossenheit: Die unterbrochene Diskantklausel ist an sich schon eine starke Überraschung, die entscheidende Pointe ist jedoch die vorenthaltene Oktavlage am Ende. Noch frappierender wirkt der Schluss des Finales von op. 33,2 (Beispiel 6).

Haydns Verfahren erinnert in der Tat an Chopins Fragment op. 41,3: auch hier der Abbruch mitten in einer Taktgruppe, und - durch die eingefügten Pausentakte extremer noch als bei Chopin - die auskomponierte formale Desorientierung.

Wäre es nun nicht angemessener, Chopins Schlüsse an klassische Verfahren anzubinden, anstatt sie als Reflex romantischer Unendlichkeitsästhetik zu sehen? - Schauen wir noch einmal genauer in die Haydnschen Beispiele: Die Offenheit, die suggeriert wird, ist primär Folge der zeitlichen Gestaltung; es geht um das Aufbrechen einer regulären Taktgruppenanordnung. Die Pausen in op. 33,2 rechnen damit, dass der Hörer musikalisch smitdenktı, den Diskurs der Musik mitverfolgt. Entscheidend ist in diesem Zusammenhang 

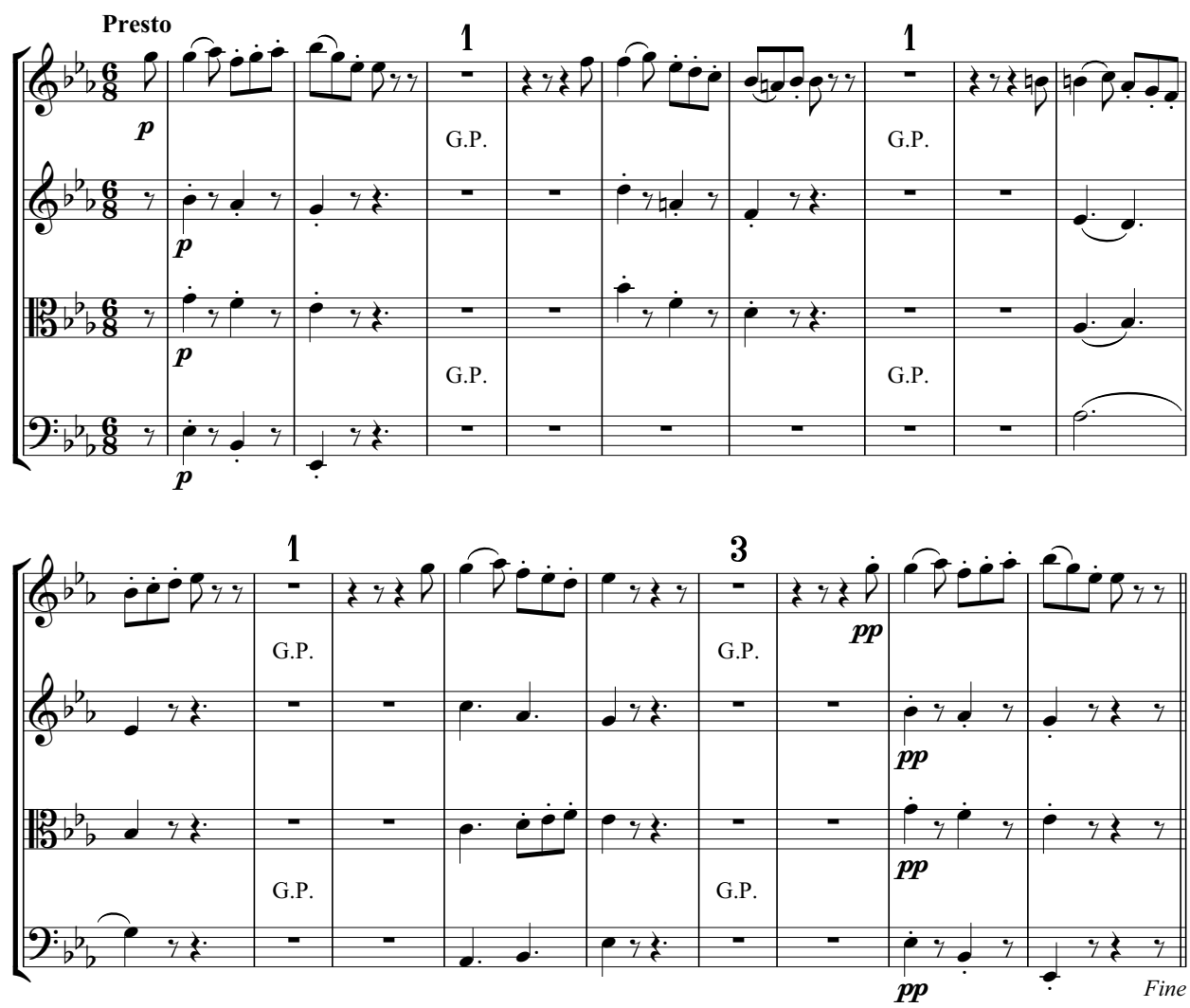

Beispiel 6: Joseph Haydn, op. 33,2, Finale, Schluss

der Affekt: Offene Schlüsse dieser Art gibt es in der Klassik zumeist in Dur-Sätzen und in Sätzen mit schnellem Tempo: Dabei geht es nicht - im romantischen Sinne - um Ironie als Verfremdung, sondern um den geistreichen `Witzı. Es ist die Irregularität des syntaktischen Verfahrens, die eine Pointe erzeugt - im vorliegenden Fall als überraschende Kontextverschiebung: Die Pause wird zunächst als Abbruch verstanden, entpuppt sich dann aber als Unterbrechung. Bei diesem Spiel ist mit dem Affekt des ıspirituoso das Lachen sozusagen mitkomponiert. Der - geistig wache - Hörer ergänzt die fehlenden Komponenten, und die Irritation wird so immer wieder eingefangen: Am Schluss bleiben dann doch ıkeine Fragen offen ‘. Chopin dagegen lässt den Hörer mit seinen offenen Schlüssen allein. Sie spiegeln die romantische Sehnsucht, die durch die Paradoxie von subjektiver Endlichkeit und objektiver Unendlichkeit provoziert wird. Diese Paradoxie wird gefühlt und nicht verstanden - in dem präzisen Sinne, in dem die romantische Philosophie das Gefühl aufgefasst hat: als ein synthetisches, dem Verstand überlegenes Vermögen. Zu diesem Zweck ist Chopin sogar bereit, die Tonalität aufzugeben, wie dies in der Mazurka op. 17,4 geschieht, die wir uns abschließend genauer ansehen. 


\section{Melancholie}

Op. 17,4: s/gelform

Ob Chopin seine Mazurkensammlungen jeweils als Zyklen gedacht hat, ist nicht gesichert, aber wahrscheinlich. Dafür spricht, dass die letzte Mazurka einer Sammlung meist besonders lang und individuell gestaltet ist. Dies gilt auch für die Mazurka op. 17,4, die als letztes und zugleich längstes Stück des Zyklus' formal insofern komplexer ist, als die übliche ABA-Form durch Einleitung, Coda und Epilog erweitert wird. Betrachten wir zunächst Beginn und Ende des Satzes:

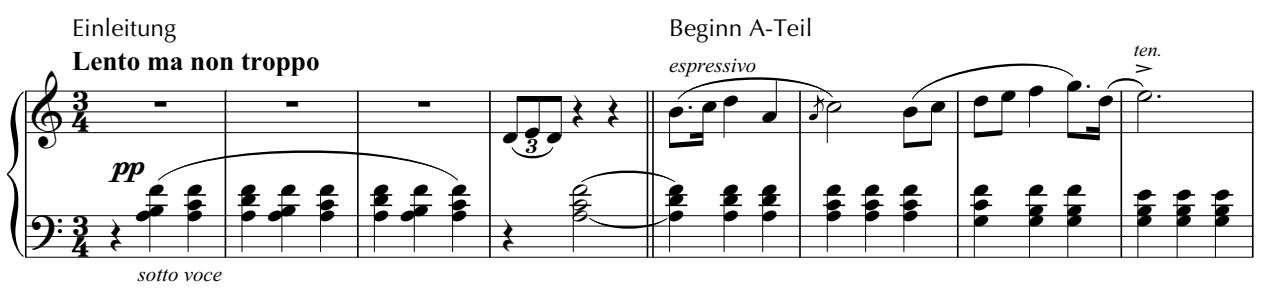

Beispiel 7a: Frédéric Chopin, Mazurka op. 17,4, Anfang

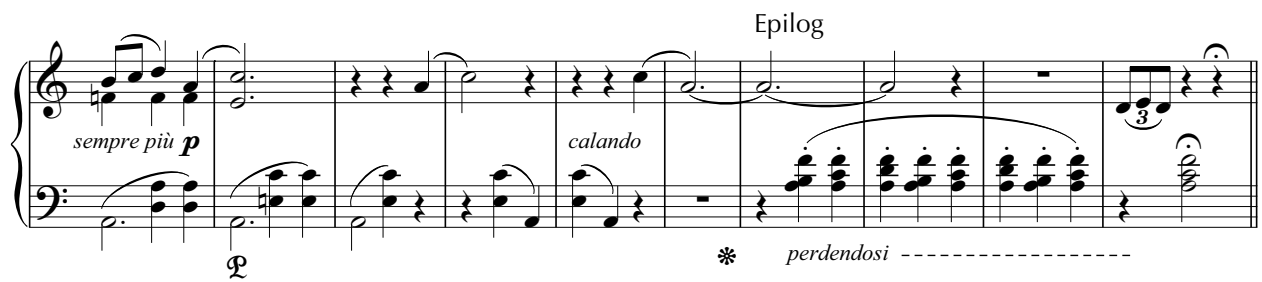

Beispiel 7b: Frédéric Chopin, Mazurka op. 17,4, Schluss

Chopin rahmt das Stück durch einen Viertakter ein, der sowohl als Eröffnungs- als auch als Schlussphrase bemerkenswert ist, da er die Tonika a-moll auslässt und stattdessen eine Harmoniefolge komponiert, die entweder als Darstellung des Sextakkords der VI. Stufe F-Dur oder als halbverminderter Septakkord der II. Stufe in Sekundakkordstellung gedeutet werden kann. Der Viertakter erzeugt somit einen bemerkenswerten Schwebezustand, der die formale Funktion ungeklärt lässt: Nimmt der Anfang den Schluss vorweg oder zitiert der Schluss den Anfang? Dazu noch einmal ein Vergleich mit Haydn, in dessen Quartetten sich Beispiele für beide Figuren finden: 
1. Der Anfang nimmt den Schluss vorweg

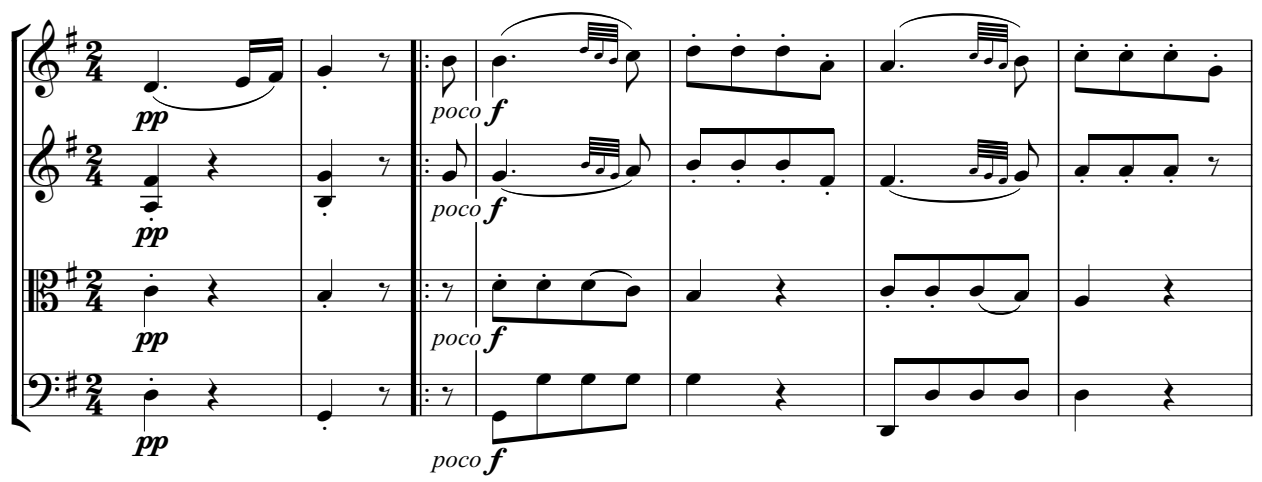

Beispiel 8a: Joseph Haydn, op. 33,5, 1. Satz, Anfang

Haydn beginnt - wieder als `Witz - den Satz mit einer Schlusskadenzierung, die den Hörer überrascht und irritiert, um dann, wenn sie am Ende des Satzes an formal korrekter Stelle erscheint, eine Pointe zu erzeugen:
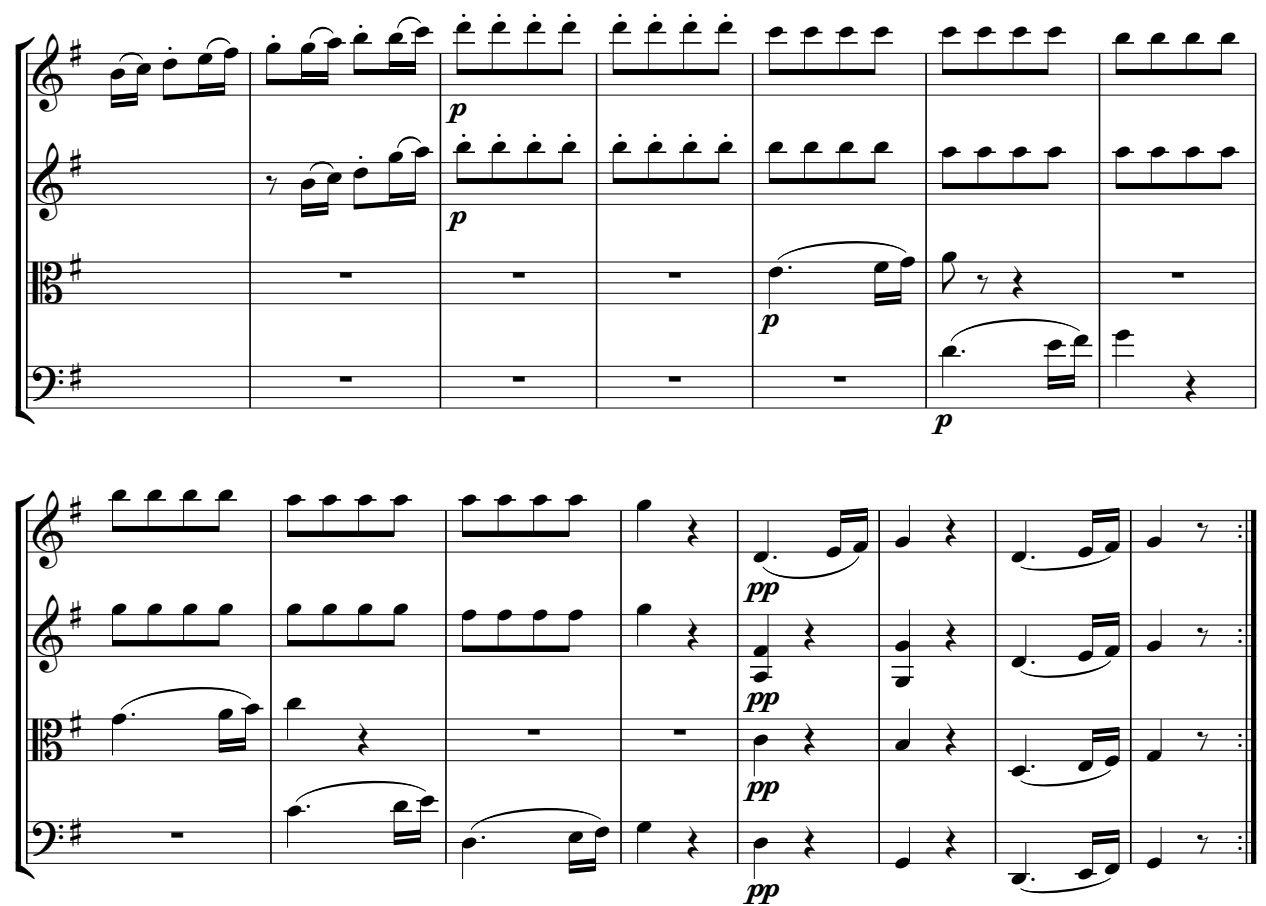

Beispiel 8b: Joseph Haydn, op. 33,5, 1. Satz, Schluss 


\section{Der Schluss zitiert den Anfang}

In dem bereits als Beispiel herangezogenen Finale aus op. 33,2 verweist dagegen umgekehrt der Schluss auf den Anfang:

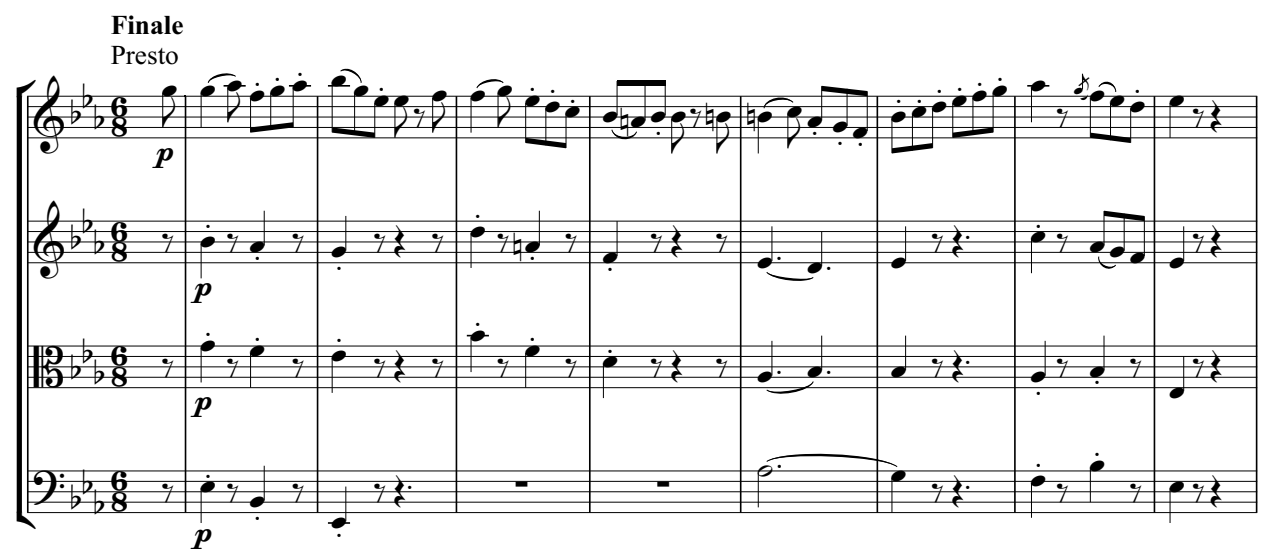

Beispiel 9: Joseph Haydn, op. 33,2, Finale, Anfang ${ }^{5}$

Chopins Viertakter ist dagegen weder öffnend noch schließend, sondern: offen. So verweist sowohl der Anfang auf das Ende, als auch das Ende auf den Anfang. Wie eine Hülle umgibt der Viertakter den Mazurkahauptteil - eine Hülle freilich, die - gleichsam perforiert - selbst keine klare Grenze, weder nach ıaußenı, noch nach sinnenı, markiert.» Op. 17,4 kann somit als Beispiel für eine Form gelten, die Charles Rosen in Anlehnung an ein Zitat von Friedrich Schlegel als »Igelform« bezeichnet hat. ${ }^{6}$ Die Form wird zum Signum einer melancholischen Stimmung, die aus dem klassischen Spiel Ernst werden lässt.

Fünf verschiedene Weisen Chopins, einen offenen Schluss zu komponieren und fünf verschiedene romantische Bilder, die dadurch evoziert werden: das auskomponierte Verklingen in op. 24,3: die Idee der `Ferne`; der Entzug des Schlussklangs in op. 30,1: die Empfindung des Mangels und der Sehnsucht; der Abbruch der Schlusstaktgruppe in op. 41,3: die Wirkung des Fragmentarischen; die sigelförmige Einrahmung in op. 17,4: eine Allegorie der Melancholie und schließlich die Spielanweisung »dal segno senza Fine« in op. 7,5 als unmittelbarer Hinweis auf das, was hinter allen Beispielen steht: das romantische Thema der Unendlichkeit. Dabei zeigt sich Chopin neben den Gebieten, auf den es ihm schon immer zugestanden wurde - Harmonik, Satztechnik und Pianistik - auch auf dem der Form als die herausragende Komponistenpersönlichkeit des frühen 19. Jahrhunderts. Dass er das Thema ıoffene Form

5 Vgl. Beispiel 6.

6 Rosen 1995, 48. 
viermusik behandelt, spiegelt auf einer Metaebene noch einmal die Dialektik von `offen und 'geschlossen`, die Paradoxie von "Systemlosigkeit, in ein System gebracht». Eine Treppenfigur, die Friedrich Schlegel gut gefallen hätte:

Jedes System ist nur Approximation seines Ideals. Die Skepsis ist ewig.

\section{Literatur}

Frank, Manfred (1989), Einführung in die frühromantische Ästhetik, Frankfurt a. M.: Suhrkamp.

Lissa, Zofia (1973), »Klavierpolonaise und -mazurka im 19. Jahrhundert», in: Gattungen der Musik in Einzeldarstellungen. Gedenkschrift Leo Schrade, hg. von Wulf Arlt u.a., Bern und München: Francke, 813-839.

Novalis (1965), Das philosophische Werk, hg. von Richard Samuel in Zusammenarbeit mit Hans-Joachim Mähl und Gerhard Schulz (= Novalis Schriften. Die Werke Friedrich von Hardenbergs, historisch-kritische Ausgabe in vier Bänden, einem Materialienband und einem Ergänzungsband in vier Teilbänden mit dem dichterischen Jugendnachlaß und weiteren neu aufgetauchten Handschriften, begründet von Paul Kluckhohn und Richard Samuel, Bd. 2), Stuttgart u. a.: Kohlhammer.

(1998), Der dichterische Jugendnachlaß (1788-1791) und Stammbucheintragungen (1791-1793), hg. von Hans-Joachim Mähl in Zusammenarbeit mit Martina Eicheldinger; Bearbeitung der Stammbücher von Ludwig Rommel (= Novalis Schriften. Die Werke Friedrich von Hardenbergs, historisch-kritische Ausgabe in vier Bänden, einem Materialienband und einem Ergänzungsband in vier Teilbänden mit dem dichterischen Jugendnachlaß und weiteren neu aufgetauchten Handschriften, begründet von Paul Kluckhohn und Richard Samuel, Bd. 6,1), Stuttgart u. a.: Kohlhammer.

Rosen, Charles (1995), The Romantic Generation, Cambridge (Mass.): Harvard University Press.

Schlegel, Friedrich (1963), Philosophische Lehrjahre 1796-1806 nebst philosophischen Manuskripten aus den Jahren 1796-1828, Erster Teil, hg. von Ernst Behler (= Friedrich Schlegel. Kritische Ausgabe seiner Werke, hg. von Ernst Behler unter Mitwirkung von Jean-Jacques Anstett und Hans Eichner, II. Abteilung: Schriften aus dem Nachlass, Bd. XVIII), Paderborn: Schöningh.

7 Schlegel 1963, 417. 